\title{
Effect of pressure on the Fermi surface and electronic structure of $\mathrm{ErGa}_{3}$
}

\author{
V. B. Pluzhnikov \\ International Laboratory of High Magnetic Fields and Low Temperatures, \\ Gajowicka 95, 53-529 Wroclaw, Poland \\ B. Verkin Institute for Low Temperature Physics and Engineering, National Academy of Sciences of Ukraine, \\ 47 Lenin Ave., Khar'kov 310164, Ukraine

\section{A. Czopnik} \\ W. Trzebiatowski Institute of Low Temperature and Structure Research, \\ P. O. Box 1410, 50-950 Wroclaw, Poland

\section{O. Eriksson} \\ Condensed Matter Theory Group, Department of Physics, University of Uppsala, \\ Box 530, S-751 21 Uppsala, Sweden \\ G. E. Grechnev and Yu. V. Fomenko \\ B. Verkin Institute for Low Temperature Physics and Engineering, National Academy of Sciences of Ukraine, \\ 47 Lenin Ave., Kharkov 310164, Ukraine \\ E-mail:grechnev@ilt.kharkov.ua \\ Received March 9, 1999
}

\begin{abstract}
The Fermi surface and cyclotron masses of the $\mathrm{ErGa}_{3}$ compound are studied by means of the de Haas-van Alphen technique under pressure. Concurrently, the electronic structure is calculated $a b$ initio for the ferromagnetic phase of $\mathrm{ErGa}_{3}$. The experimental data have been analyzed on the basis of the calculated volume-dependent band structure and compared with available results for isostructural $\mathrm{TmGa}_{3}$ and $\mathrm{LuGa}_{3}$ compounds.
\end{abstract}

PACS: 71.18.+y, 71.20.Eh, 71.70.Gm

\section{Introduction}

The objective of the present work is a study of the pressure effect on the Fermi surface (FS) and cyclotron masses of the $\mathrm{ErGa}_{3}$ compound by means of the de Haas-van Alphen (dHvA) effect. The pressure derivatives of $\mathrm{dHvA}$ frequencies and cyclotron masses are of particular interest due to their assumed sensitivity to details of the exchange interaction and many-body effects. Therefore, the present investigation can provide a critical test for recently developed methods of $a b$ initio calculations of electronic and magnetic structures, and to stimulate the formulation of improved theories for rareearths (RE).

This work represents an extension of our recent studies [1-3] of the FS and electronic structure in the cubic $\mathrm{REGa}_{3}$ compounds at ambient pressure.
There are very little data available on the physical properties of $\mathrm{ErGa}_{3}$. The compound crystallizes in the $\mathrm{AuCu}_{3}$-type cubic structure and orders antiferromagnetically at $T_{N}=2.8 \mathrm{~K}$ by means of a continuous transition, and the corresponding magnetic structure is presumably the incommensurate modulated one [4]. It can be expected that at low temperatures $\mathrm{ErGa}_{3}$ reveals large and field-dependent magnetization, in the same manner as is the case of $\mathrm{TmGa}_{3}$ [2]. This provides a number of complications in the Fourier analysis of dHvA oscillations. Specifically, the dHvA effect has to be studied in sufficiently strong magnetic fields where magnetization is almost saturated. These fields are expected to be higher than the critical field destroying the antiferromagnetic order. By this means the dHvA effect can be investigated in a paramagnetic 
phase of $\mathrm{ErGa}_{3}$, in which magnetic field leads to a quasi-ferromagnetic configuration of magnetic moments.

In the present work the experimental study of the dHvA effect under pressure is complemented by $a b$ initio calculations of the spin-polarized electronic structure of $\mathrm{ErGa}_{3}$ when the atomic volume is varied through a small range around the equilibrium value. Basically, the dHvA data supplemented by results of the calculations provide a possibility to estimate the volume dependencies of the FS and exchange interaction parameters, as well as the many-body enhancement of band cyclotron masses. A comparison of the data of the pressure effect and the calculated volume dependent band structure can be especially useful in testing the adequacy of theoretical models employed for rare-earth compounds. The evaluated parameters of the electronic structure of $\mathrm{ErGa}_{3}$ are also compared with corresponding results obtained for the isostructural compounds $\mathrm{TmGa}_{3}$ and $\mathrm{LuGa}_{3}$ at ambient pressure $[1,2]$.

\section{Experimental techniques}

Single crystals of $\mathrm{ErGa}_{3}$ were grown by the flux method from the melt of the nominal composition $90 \%$ at. Ga and $10 \%$ at. Er. The purity of starting metals was $6 \mathrm{~N}$ for $\mathrm{Ga}$ and $4 \mathrm{~N}$ for Er. The feed placed in a alumina crucible and sealed in a quartz tube in argon atmosphere under pressure of 150 Torr at room temperature, was heated in a resistance furnace up to $920{ }^{\circ} \mathrm{C}$, held at this temperature for $48 \mathrm{~h}$ and then slowly cooled down at the rate $0.8 \mathrm{~K} / \mathrm{h}$. The synthesis was stopped at about $350{ }^{\circ} \mathrm{C}$ and then sample was cooled rapidly down to room temperature for avoiding the formation of $\mathrm{ErGa}_{6}$ in peritetic reaction [5]. The resulting crystals of $\mathrm{ErGa}_{3}$ were immersed in an excess of Ga which is easy to remove. The obtained crystals had the form of cubes with maximum dimensions $5 \times 5 \times 5 \mathrm{~mm}$. According to the $\mathrm{x}$-ray examination the quality of single crystals was very good.

The dHvA effect measurements were performed on a spherical sample (diameter $2.5 \mathrm{~mm}$ ) by using a standard field modulation technique at temperatures down to $1.5 \mathrm{~K}$ and in magnetic fields up to $15 \mathrm{~T}$ applied along principal crystallographic axes. Large amplitudes of the observed dHvA oscillations can be considered as another prove of the high quality of $\mathrm{ErGa}_{3}$ single crystals. A standard $\mathrm{Cu}-\mathrm{Be}$ clamp was used for the pressure effect study with an extracted benzine solvent as the medium transmitting pressure to the sample. The maximum pressure employed was $6.4 \mathrm{kbar}$ at $4.2 \mathrm{~K}$. A small manganin coil with resistance about $60 \Omega$ was placed near the sample to measure the applied pressure. Preliminary this coil has been trained to cooling-pressure and then calibrated by measuring the superconducting transition temperature of Sn [6]. A deviation of the manganin coil resistance due to the residual magnetic field of the superconducting magnet has been also taken into account. The sample, the pick-up coil, and the manganin coil, all were placed in a teflon cell, filled with the extracted benzine solvent, and then the cell was put in the pressure clamp. A deviation from hydrostatic pressure and its effect on the measurements are estimated to be negligible by observing that the superconducting transition width of Sn does not change noticeably and amplitudes of the dHvA oscillations do not decrease substantially under pressures used in this work.

Since the pressure clamp is heated with the modulation field, there is a difference in temperatures between the helium bath and the sample in the pressure clamp. The modulation amplitude and frequency used in measurements were $40 \mathrm{G}$ and $38.5 \mathrm{~Hz}$, respectively, These amplitude and frequency were chosen to produce a large enough dHvA signal, and, at the same time, to reduce the heating power, which leads to a temperature difference not exceeding $0.02 \mathrm{~K}$.

In a magnetic material, the $\mathrm{dHvA}$ oscillations are periodic in $B^{-1}$, where $B$ is the magnetic induction. For a spherical sample as we have used $\mathbf{B}=\mathbf{H}_{\text {appl }}{ }^{+}$ $+(8 \pi / 3) \mathbf{M}$, where $\mathbf{H}_{\text {appl }}$ is the applied field and $\mathbf{M}$ is the magnetization. Complementary magnetization measurements were performed by a home made vibrating sample magnetometer. The field dependence of the magnetization along the principal crystallographic axes is shown in Fig. 1. This dependence can be reproduced by a fitting calculation in the molecular-field approximation. The Hamiltonian employed contains the crystal field (CF), the exchange and Zeeman terms. The molecular-field exchange parameter was estimated, based on the value of the paramagnetic Curie temperature, $\Theta_{p}=$ $=-10 \mathrm{~K}$. Then the best fit to the experimental data was obtained for the CF parameters $x$ and $W$ (in the usual notations of Ref. [7]) equal to 0.22 and $0.25 \mathrm{~K}$, respectively. This gives the $\Gamma_{7}$ doublet as the $\mathrm{CF}$ ground state, and the first excited state appeared to be the $\Gamma_{8}^{(1)}$ quartet at about $30 \mathrm{~K}$. The total CF splitting is about $120 \mathrm{~K}$. These parameters provided a possibility for evaluating the angular dependence of the magnetization [3].

The applied pressure modifies a magnitude and field dependence of the magnetization due to a 


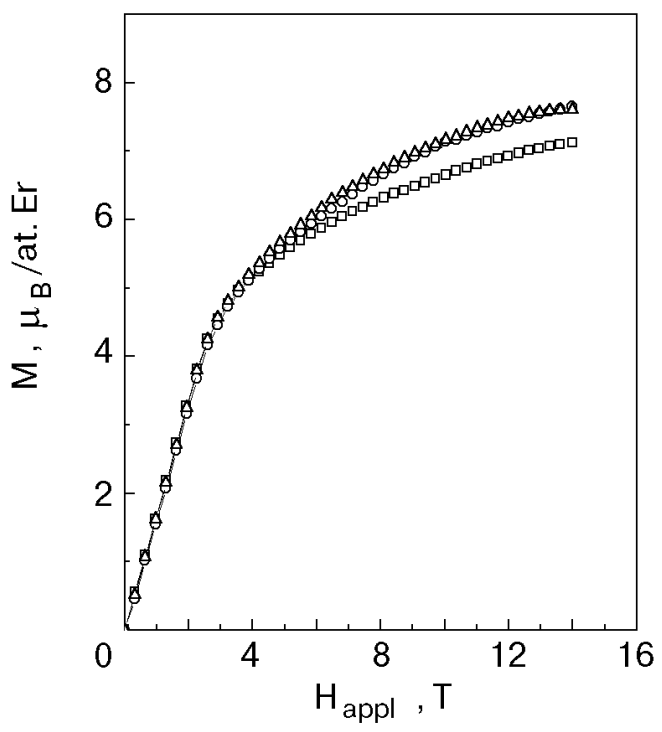

Fig. 1. Magnetization of $\mathrm{ErGa}_{3}$ at $1.7 \mathrm{~K}$ in the magnetic field applied along $\langle 100\rangle(\square),\langle 110\rangle(O)$, and $\langle 111\rangle(\Delta)$ axes.

pressure influence on the CF splitting, as well as on the exchange interaction. It is well known, that CF of metallic rare-earth compounds contains contributions from charges of surrounding ligands as well as from the direct Coulomb and exchange interactions of the rare-earth ion with conduction electrons. In order to estimate the influence of the pressure on the $\mathrm{CF}$ we have restricted ourselves to the contribution from surrounding ligands within the point charge model. The applied pressure $P$ brings about the volume dilatation $\delta V / V=-P / c_{B}$, were $c_{B}-$ bulk modulus. Under pressure of $10 \mathrm{kbar} \delta V / V$ is estimated to be -0.013 , when we assume for $\mathrm{ErGa}_{3}$ the bulk modulus of $\mathrm{TmGa}_{3}$, equal to $c_{B}=765 \mathrm{kbar}$ [8]. The change of $\mathrm{CF}$ due to this dilatation causes a variation of the magnetic induction at $1.7 \mathrm{~K}$ in the applied field of $15 \mathrm{~T}$ not larger than $20 \mathrm{G}$. One can estimate also a change of the magnetization in $\mathrm{ErGa}_{3}$ due to a variation of the exchange interaction parameter under pressure by making use of appropriate data obtained for the isostructural $\mathrm{REIn}_{3}$ compounds $[9,10]$. The corresponding variation of the magnetic induction with the applied pressure of $10 \mathrm{kbar}$ is about $-10 \mathrm{G}$ at $1.7 \mathrm{~K}$ in the field of $15 \mathrm{~T}$. Therefore, the total change of the magnetic induction reaches only $10 \mathrm{G}$, giving the relative variation of the $\mathrm{dHvA}$ frequency $\delta F / F \simeq$ $\simeq 2 \cdot 10^{-4} \mathrm{kbar}^{-1}$, which may be neglected in the Fourier analysis of the dHvA oscillations.

The dHvA effect measurements were carried out in magnetic fields higher than $8 \mathrm{~T}$ where the magnetization does not change appreciably and the Fourier analysis of the dHvA oscillations can be performed. In another case a dHvA frequency

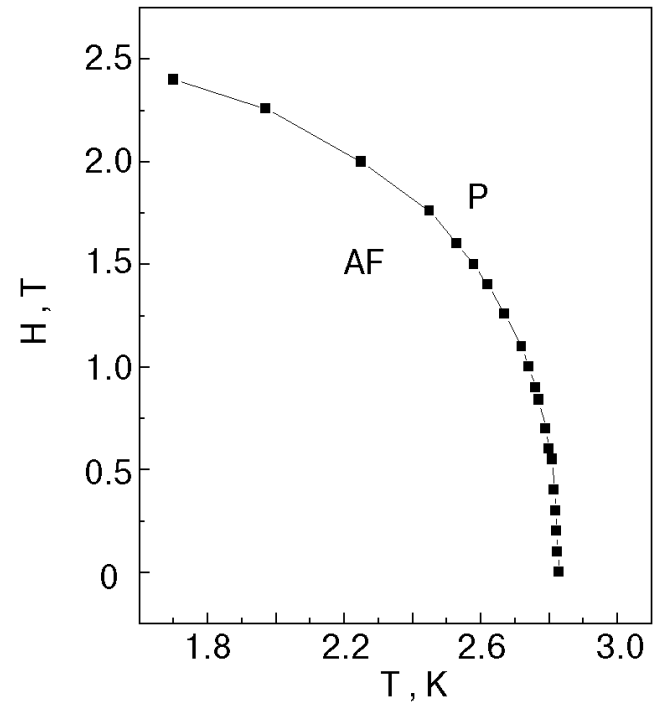

Fig. 2. Sketch of the magnetic phase diagram of $\mathrm{ErGa}_{3}$ in the magnetic field applied along the $\langle 100\rangle$-axis. The curve is a line of phase transitions between an antiferromagnetic state and the paramagnetic one.

would change its value following the strength of external magnetic field. It turns out that for this intensity range $\mathrm{ErGa}_{3}$ is in the paramagnetic state, and the measurements were carried out in this phase well above the antiferro-paramagnetic transition. Actually, along all principal crystallographic axes, $\langle 001\rangle,\langle 011\rangle$, and $\langle 111\rangle$ the critical field of the antiferromagnetic - paramagnetic transition does not exceed $3 \mathrm{~T}$. This is evident from a sketch of the magnetic phase diagram in Fig. 2, where the curve represents a transition between an antiferromagnetic state and the paramagnetic one for the fields applied along $\langle 100\rangle$ axes. Actually, the magnetic phase diagram of $\mathrm{ErGa}_{3}$ is more complex, and its full description, together with neutron diffraction data and the CF scheme examination, will be published elsewhere [11].

The magnetization in magnetic fields higher than $8 \mathrm{~T}$ tends to saturate and magnetic moments settle into a quasi-ferromagnetic configuration. Moreover, the magnetization along all directions in a magnetic field higher than $8 \mathrm{~T}$ appeared to be almost temperature independent in the range 1.7-4.2 K. Therefore, the evaluated values of the magnetization along the principal crystallographic axes were used in the Fourier analysis of dHvA oscillations. It should be pointed out that for the field induced quasi-ferromagnetic configuration the dHvA spectrum of $\mathrm{ErGa}_{3}$ can be compared with results of band structure calculations for the corresponding spinpolarized state. 


\section{Details of calculations}

At present it is commonly believed [12-14] that, within the local spin-density approximation (LSDA), a strict band treatment of the $4 f$ states is inadequate for heavy rare earths. In the corresponding spin-polarized calculations the $f$-shell is not filled and the $4 f$ bands, which act as a sink for electrons, always cut the Fermi level $\left(E_{F}\right)$ leading to absurd values of specific heat coefficients [12] and wrong $4 f$ occupancies, close to the divalent (i.e., atomic) configuration [15].

According to the photoemission data [15-17], the $4 f$ spectral density for Er and Er-based compounds was observed about $5 \mathrm{eV}$ below $E_{F}$. Therefore, for the present purpose, which is mainly to describe the band structure for the ground state near $E_{F}$, it seems reasonable to treat $4 f$ states in $\mathrm{ErGa}_{3}$ as semilocalized core states, in line with Refs. [10,13,14,18].

In fact, the standard rare-earth model [12] is employed in this work in the limit of the large Hubbard repulsion $U$ within the $a b$ initio LSDA scheme [19] for the exchange-correlation effects. The localized $f$-states of Er were treated as spin-polarized outer-core wave functions, contributing to the total spin density. Consequently, the spin occupation numbers were fixed by applying the RusselSaunders coupling scheme to the $4 f$ shell, which was not allowed to hybridize with conduction electrons.

The self-consistent band structure calculations were carried out for the paramagnetic configuration phase of $\mathrm{ErGa}_{3}$ by using the linear muffin-tin orbital method (LMTO) in the atomic sphere approximation (ASA) with combined corrections to ASA included [20,21]. In the framework of the LSDA, the spin density of the $4 f$ states polarizes the «spin-up» and «spin-down» conduction electron states through the local exchange interaction. The exchange split conduction electron states interact with the localized $f$ states at other sites, appearing as the medium for the indirect $f-f$ interaction $[10,18]$.

In order to calculate FS orbits, the charge densities were obtained by including spin-orbit coupling at each variational step, as suggested in Refs. $[12,13]$. In this case the spin is no longer a good quantum number, and it is not possible to evaluate the electronic structure for «spin-up» and «spindown» bands separately. Also, we have employed the approximation, which has been extremely successful for rare-earths [12], namely, to omit spin-orbit coupling in spin-polarized band structure calculations for $\mathrm{ErGa}_{3}$. It gives the possibility to

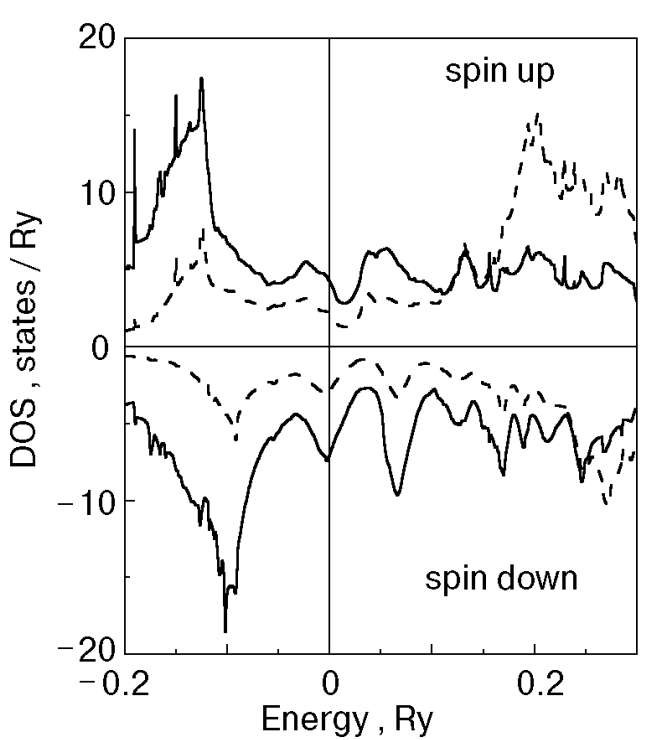

Fig. 3. Partial densities of states as a function of energy (relative to the Fermi energy $E_{F}$ ) for the ferromagnetic phase of $\mathrm{ErGa}_{3}$. Solid lines stand for $p$-states of $\mathrm{Ga}$, and dashed lines represent $d$-states of Er.

elucidate the role of the spin-orbit coupling, and also to present «spin-up» and «spin-down» bands for field-induced ferromagnetic phase of $\mathrm{ErGa}_{3}$, where the exchange splitting is much larger than the spin-orbit coupling.

The band structure calculations were performed self-consistently on a uniform mesh of 455 points in the irreducible wedge of the cubic Brillouin zone for a number of lattice parameters close to the experimental one. The individual atomic radii of the components were chosen following the method outlined in Ref. [22].

The results obtained with the LMTO-ASA method were verified and supplemented by carrying out ab initio relativistic full-potential LMTO calculations of the electronic structure of $\mathrm{ErGa}_{3}$ in an external magnetic field with the Zeeman term included (details of this method have been described elsewhere [23]). The calculated density of states (DOS) and band structure along selected high symmetry lines appeared to be similar in the two types of calculations.

The calculated partial densities of states for the ferromagnetic phase of $\mathrm{ErGa}_{3}$ are presented in Fig. 3. Two fairly broad peaks, associated with bonding and antibonding states, originate due to hybridization of $5 d$-states of Er and $4 p$-states of Ga. As can be seen in Fig. 3, these $p$-states give substantial contribution to the total DOS at the Fermi energy. On the other hand, the exchange splitting is more pronounced for the $5 d$-states of $\operatorname{Er}$ (about $20 \mathrm{mRy}$ ) due to the local exchange interaction. For hybri- 
dized electronic states at $E_{F}$ the exchange splitting takes smaller values, reducing to about $1 \mathrm{mRy}$ for the pure $p$-states.

\section{Results and discussion}

The Fourier spectra of dHvA oscillations in $\mathrm{ErGa}_{3}$ observed along $\langle 100\rangle$ axis at different pressures are presented in Fig. 4, and the pressure effect on the corresponding dHvA frequencies is exhibited in Fig. 5. For all the principal crystallographic axes, $\langle 100\rangle,\langle 110\rangle$, and $\langle 111\rangle$, the dHvA frequencies at ambient pressure and their pressure derivatives $d \ln F / d P$ are listed in Table 1 .

Table 1

DHvA frequencies at ambient pressure, their pressure derivatives, and cyclotron effective masses at ambient pressure in $\mathrm{ErGa}_{3}$

\begin{tabular}{c|c|c|c|c}
\hline \hline $\begin{array}{c}\text { Field } \\
\text { direction }\end{array}$ & $F, \mathrm{MG}$ & \multicolumn{2}{|c|}{$d \ln F / d P, 10^{3} \mathrm{kbar}^{-1}$} & $m_{c}^{*}, m_{0}$ \\
\cline { 2 - 5 } & experiment & experiment & theory & experiment \\
\hline \hline$\langle 100\rangle$ & 98.71 & $2.3 \pm 0.3$ & 1.3 & $0.96 \pm 0.02$ \\
& 97.81 & $1.4 \pm 0.4$ & 1.1 & - \\
& 41.07 & $1.7 \pm 0.2$ & 2.0 & $0.91 \pm 0.02$ \\
& 30.27 & $0.36 \pm 0.02$ & - & $0.92 \pm 0.03$ \\
& 12.66 & $-2.7 \pm 0.1$ & -2.8 & $0.44 \pm 0.02$ \\
& 4.35 & - & - & $0.55 \pm 0.02$ \\
$\langle 110\rangle$ & 96.03 & $1.2 \pm 0.2$ & 1.2 & $0.89 \pm 0.02$ \\
& 95.17 & $1.0 \pm 0.3$ & 1.1 & - \\
& 15.80 & $-1.2 \pm 0.1$ & - & - \\
& 15.14 & $-1.1 \pm 0.1$ & - & $0.57 \pm 0.02$ \\
& 11.95 & $-2.4 \pm 0.2$ & -2.0 & $0.84 \pm 0.04$ \\
& 3.37 & $-4.5 \pm 0.3$ & - & $0.28 \pm 0.02$ \\
$\langle 111\rangle$ & 88.30 & $2.5 \pm 0.3$ & 1.4 & $0.80 \pm 0.02$ \\
& 87.58 & $1.7 \pm 0.1$ & 1.2 & - \\
& 35.47 & $2.3 \pm 0.2$ & 1.9 & $0.70 \pm 0.02$ \\
& 4.21 & - & - & $0.51 \pm 0.02$ \\
\hline \hline
\end{tabular}

For reference, the angular dependence of dHvA frequencies in the $(100)$ and $(110)$ planes, obtained at ambient pressure in Ref. 3, is shown in Fig. 6. The intersections of the calculated FS of $\mathrm{ErGa}_{3}$ with planes of the cubic Brillouin zone (Fig. 7) reveal the «spin-split» almost spherical electron FS centered at $R$-point and the complicated multiply connected hole FS centered at $\Gamma$ - and $X$-points. As can be seen in Fig. 6, the agreement between the calculated FS and the experimental data is quite good in the range of the high dHvA frequencies (branch $a$ which arise from the FS sheet at $R$-point, and branch $d$, associated with the largest sheet of the hole FS centered at $\Gamma$-point), as well as the medium ones (branch $b$, related to the hole FS sheet at $X$-point). Instead of the calculated branch $j$,

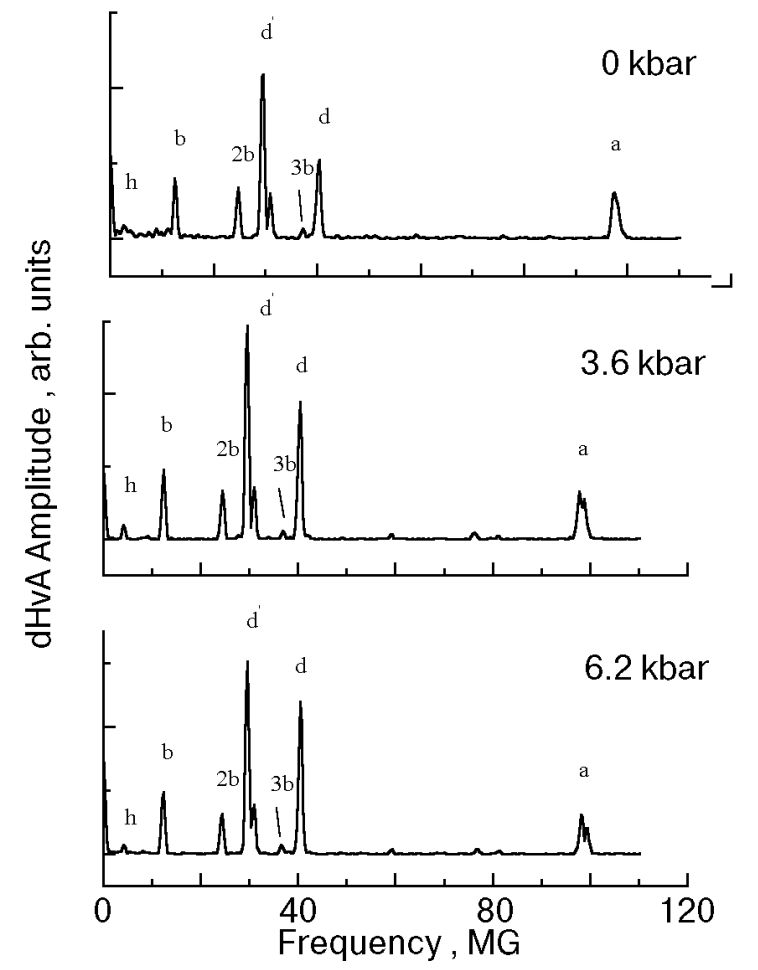

Fig. 4. Fourier spectra of dHvA oscillations observed at $2.1 \mathrm{~K}$ for magnetic fields directed along [100] axis at ambient pressure, $3.6 \mathrm{kbar}$, and $6.2 \mathrm{kbar}$.

which arise from a «neck» at the symmetry line $\Gamma R$, a branch with a different angular field dependence has been observed in the range of low dHvA frequencies. It is analogous to the branch previously found in $\mathrm{LuGa}_{3}$ [1], and labelled $h$ in Fig. 6 .

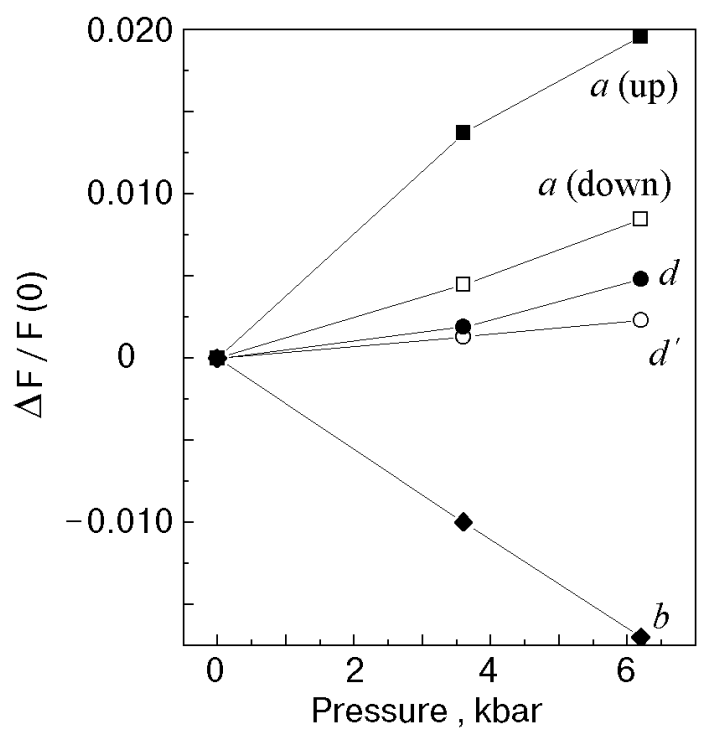

Fig. 5. Fractional changes of the dHvA frequencies, $\Delta F / F(0)$, as a function of pressure for the [100] magnetic field direction at $2.1 \mathrm{~K}$. The frequencies are labelled according to Ref.[3] and Fig. 6. The $a(\mathrm{up})$ and $a$ (down) refer to the oscillations originating from the «spin-up» and «spin-down» subbands of the $a$ branch, respectively. The solid lines are guides for the eye. 


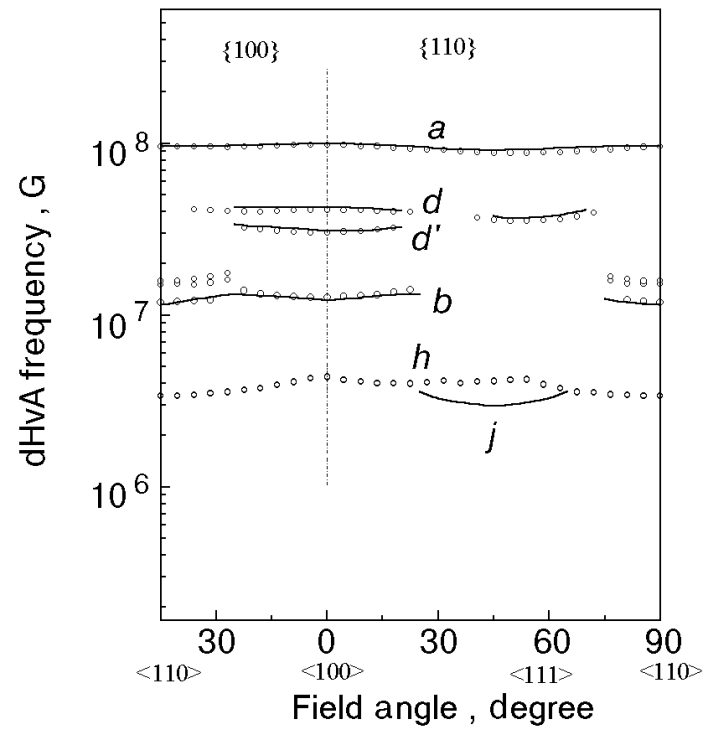

Fig. 6. Angular dependence of the dHvA frequencies in $\mathrm{ErGa}_{3}$, taken from Ref. [3]. Circles stand for the experimental data, solid lines show calculated results.

Basically, the dHvA spectrum of $\mathrm{ErGa}_{3}$ in the field-induced ferromagnetic configuration appeared to be close to the spectrum of $\mathrm{LuGa}_{3}$ [1], except for the presence of additional branch $d^{\prime}$ that is just below the $d$ branch and located in the vicinity of the $\langle 100\rangle$ axis. It should be pointed out that in a ferromagnetic configuration phase of the isostructural $\mathrm{TmGa}_{3}$ compound the $\mathrm{dHvA}$ spectrum contains several $h$-like branches in the low frequency range and no $d^{\prime}$-branch [2].

The dHvA oscillations originating from the «spin-split» subbands were observed only for the $a$ branch. This splitting is seen in Fig. 4 and Fig. 5, but not resolved in Fig. 6 owing to the scale chosen. Based upon the calculated partial DOS in Fig. 3, one can expect the more easily observed dHvA

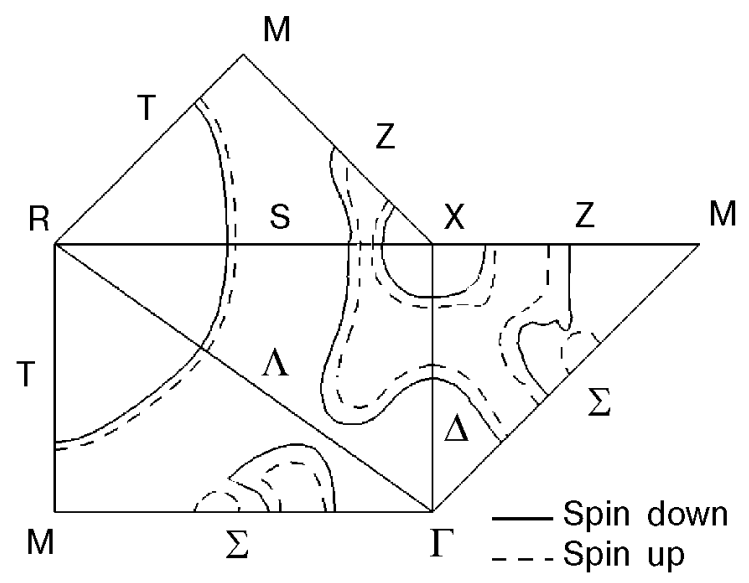

Fig. 7. Intersection of the Fermi surface for $\mathrm{ErGa}_{3}$ with the Brillouin zone planes. oscillations related to the majority states, having lower DOS and, correspondingly, lower band masses. In fact, the frequencies calculated for «spin-up» bands (which are actually presented in Fig. 6) appeared to be in better agreement with the experimentally observed ones.

The experimental pressure derivatives $d \ln F / d P$, presented in Table 1, are rather large in comparison with free-electron scaling prediction, which gives two-thirds the volume compressibility or $0.87 \cdot 10^{-3} \mathrm{kbar}^{-1}$, provided the available bulk modulus of $\mathrm{TmGa}_{3}$ [8] is accepted for $\mathrm{ErGa}_{3}$. Basically, there is a qualitative agreement between the experimental and calculated $d \ln F / d P$ derivatives (again, the bulk modulus of $\mathrm{TmGa}_{3}$ was used to convert the calculated volume derivatives to the pressure ones, listed in Table 1). The main discrepancy between these derivatives can be seen for the $a$ branch, where the average exchange splitting is given by

$$
\Delta E=2 \mu_{B} \Delta F\left(m_{0} / m_{c}^{b}\right),
$$

where $m_{c}^{b}$ is the calculated band cyclotron mass and $m_{0}$ is the free-electron mass. Taking into account the small value of this exchange splitting for the $a$ branch (about $1 \mathrm{mRy}$ ), the difference between experimental pressure derivatives for the corresponding «spin-split» subbands can be considered as surprisingly large.

It was originally suggested in Ref. [24], that there are two contributions in ferromagnetic metals to the pressure derivative of $\mathrm{dHvA}$ frequency. The first («potential») contribution comes from an atomic volume effect on the crystal potential, and also from a scaling effect due to the change of the Brillouin zone size. It can be approximated by the corresponding derivative for a non-magnetic reference compound with a close value of the compressibility (in our case it can be $\mathrm{LuGa}_{3}$ ). The second («magnetic») contribution originates from the redistribution of conduction electrons between spinsplit subbands with pressure and the corresponding changes of volume enclosed by FS sheets. Within the Stoner-Wohlfarth model this contribution for ferromagnetic $3 d$-metals was described qualitatively by considering a pressure effect on the exchange splitting $\Delta$ [24]. According to the present calculations, Fermi surfaces of $\mathrm{ErGa}_{3}$ do not change uniformly because of a strong $\mathbf{k}$-dependent $p$ - $d$ mixing effect on the exchange-split conduction band. As a result, the difference between pressure derivatives of the spin-split FS cross-sectional areas is inconsistent with prediction based on the Stoner-Wohlfarth model. 
At this point, it is difficult to explain the discrepancy between the experimental and calculated derivatives of dHvA frequencies for the spin-split $a$ branch in $\mathrm{ErGa}_{3}$. A detailed consideration of this problem should obtain further arguments in future planned experiments, which are aimed to reveal exchange-splitting for other FS sheets, as well as their pressure dependence. Also, a study of the dHvA effect under pressure in the reference nonmagnetic compound $\mathrm{LuGa}_{3}$ will provide a possibility to evaluate the «potential» contributions to the pressure derivatives for corresponding FS cross-sections of $\mathrm{ErGa}_{3}$. In addition, spin-polarized calculations of the volume-dependent FS with full-potential LMTO method will be helpful to clarify this problem. Compared to the LMTO-ASA method used for this purpose, a full-potential technique provides better description of electronic density in the interstitial region and, accordingly, more realistic $p$-like and $p$ - $d$ hybridized conduction electron states.

Cyclotron masses $m_{c}^{*}$ have been determined at ambient pressure for all dHvA frequencies in the field applied along the $\langle 100\rangle,\langle 110\rangle$ and $\langle 111\rangle$ axes, and appeared to be smaller than free electron mass (see Table 1). Also, the cyclotron masses measured at a pressure of $4 \mathrm{kbar}$ for branches $a$ and $d$ in the field applied along the $\langle 111\rangle$ axis are presented in Table 2. Band cyclotron masses $m_{c}^{b}$ have been calculated for $a$ and $d$ branches, and are also given in Table 2 .

The mass enhancement factor $\lambda$, which is defined by relation $m_{c}^{*}=m_{c}^{b}(1+\lambda)$, presents a measure of interaction strength of conduction electrons with low energy excitations. The $\lambda$-factors for electrons on $a$ and $d$ orbits in $\mathrm{ErGa}_{3}, \mathrm{TmGa}_{3}$ [2], and $\mathrm{LuGa}_{3}[1,2]$ are listed in Table 2. In non-magnetic
$\mathrm{LuGa}_{3}$ the $\lambda$-factor is presumably a measure of electron-phonon interactions, whereas in $\mathrm{ErGa}_{3}$ and $\mathrm{TmGa}_{3}$ this factor also contains contribution(s) coming from magnetic excitations. As seen in Table 2 , in $\mathrm{LuGa}_{3}$ the $\lambda$ factor ranges from 0.3 ( $d$ branch) to about 1 ( $a$ branch). Assuming that values of $\lambda_{e-\mathrm{ph}}$ in $\mathrm{REGa}_{3}$ are close to the corresponding ones in $\mathrm{LuGa}_{3}$, one can estimate magnetic contributions $\lambda_{\text {mag }}$ in $\mathrm{ErGa}_{3}$ to be $0.4-0.6$ and 0.4-0.7 for $a$ and $d$ orbits, respectively. In $\mathrm{TmGa}_{3}$ the corresponding values of $\lambda_{\text {mag }}$ are larger and more anisotropic, namely $0.5-1$ and $0.8-1.5$.

The hybridization of conduction electrons with If states could contribute to the large cyclotron masses observed in $\mathrm{ErGa}_{3}$ (and $\mathrm{TmGa}_{3}$ ), and affect the shape of FS as well. For heavy rare-earths these hybridization effects are commonly overestimated within LSDA, and corresponding calculations would lead to substantial reduction of the conduction band width in $\mathrm{REGa}_{3}$ and, therefore, to remarkably different bulk properties in comparison to $\mathrm{LuGa}_{3}$. In accord with the lattice parameters behavior, which decrease slightly in a linear fashion in the series $\mathrm{ErGa}_{3}, \mathrm{TmGa}_{3}$, and $\mathrm{LuGa}_{3}$ due to the lanthanide contraction, it can be expected, however, that conduction band widths are close in $\mathrm{REGa}_{3}$, and band cyclotron masses should be also close. At the present stage even calculations performed within LSDA+U or a Hubbard-like scheme would not be of decisive importance [14], and more elaborated analysis is necessary to estimate a scale of the hybridization effects.

One may assume that the distinctions between effective masses of $\mathrm{TmGa}_{3}$ and $\mathrm{ErGa}_{3}$ are most likely due to the different ground states ${ }^{3} \mathrm{H}_{6}$ and ${ }^{4} I_{15 / 2}$ multiplets of $\mathrm{Tm}^{3+}$ and $\mathrm{Er}^{3+}$ ions in the $\mathrm{CF}$ of $\mathrm{TmGa}_{3}$ and $\mathrm{ErGa}_{3}$, respectively. The CF scheme

Table 2

Cyclotron masses (experimental, $m_{c}^{*}$, and calculated, $m_{c}^{b}$, in units of free-electron mass) and the corresponding mass enhancement factor $\lambda$ in $\mathrm{REGa}_{3}$ compounds at ambient pressure. For $\mathrm{ErGa}_{3}$, two effective masses measured at 4 kbar are also presented

\begin{tabular}{|c|c|c|c|c|c|c|c|c|c|c|}
\hline \multirow{2}{*}{$\begin{array}{c}\text { Branch } \\
\text { and } \\
\text { orientation }\end{array}$} & \multicolumn{4}{|c|}{$\mathrm{ErGa}_{3}$} & \multicolumn{3}{|c|}{$\mathrm{TmGa}_{3}{ }^{\mathrm{a}}$} & \multicolumn{3}{|c|}{$\mathrm{LuGa}_{3}{ }^{\mathrm{a}}$} \\
\hline & $m_{c}^{*}$ & $m_{c}^{*}, 4 \mathrm{kbar}$ & $m_{c}^{b}$ & $\lambda$ & $m_{c}^{*}$ & $m_{c}^{b}$ & $\lambda$ & $m_{c}^{*}$ & $m_{c}^{b}$ & $\lambda$ \\
\hline $\bar{e} a,\langle 100\rangle$ & $0.96 \pm 0.02$ & - & 0.40 & 1.4 & $1.20 \pm 0.02$ & 0.41 & 1.93 & $0.74 \pm 0.02$ & 0.38 & 0.95 \\
\hline$a,\langle 110\rangle$ & $0.89 \pm 0.02$ & - & 0.37 & 1.4 & $1.02 \pm 0.03$ & 0.38 & 1.68 & $0.73 \pm 0.02$ & 0.36 & 1.03 \\
\hline$a,\langle 111\rangle$ & $0.80 \pm 0.02$ & $0.94 \pm 0.03$ & 0.37 & 1.16 & $0.77 \pm 0.02$ & 0.38 & 1.03 & $0.57 \pm 0.02$ & 0.36 & 0.58 \\
\hline$d,\langle 100\rangle$ & $0.91 \pm 0.02$ & - & 0.46 & 0.98 & $1.30 \pm 0.02$ & 0.46 & 1.83 & $0.63 \pm 0.02$ & 0.48 & 0.31 \\
\hline$d,\langle 111\rangle$ & $0.70 \pm 0.02$ & $0.92 \pm 0.03$ & 0.40 & 0.75 & $0.90 \pm 0.02$ & 0.42 & 1.14 & $0.53 \pm 0.02$ & 0.39 & 0.36 \\
\hline
\end{tabular}

a Taken from Ref. [2] 
of $\mathrm{TmGa}_{3}$ provides the triplet $\Gamma_{5}^{(1)}$ as the ground state with intrinsic magnetic and quadrupolar moments [8]. Therefore, in $\mathrm{TmGa}_{3}$ the enhancement factor $\lambda$ presumably contains a contribution from coupled magnetic-quadrupolar excitations. Also, in the quasi-ferromagnetic configuration of magnetic moments, the exchange splitting of conduction bands can vary in $\mathrm{ErGa}_{3}$ and $\mathrm{TmGa}_{3}$ due to the difference in corresponding $4 f$-shell spin occupation numbers. At the moment one cannot attribute the differences in cyclotron masses (and angular dependencies of $\mathrm{dHvA}$ frequencies as well) in an unambiguous way in $\mathrm{ErGa}_{3}$ and $\mathrm{TmGa}_{3}$ either to the conduction band splitting or the magnetostriction.

In addition, one more mechanism can govern these differences. Namely, it was shown in Ref. [25] that virtual magnetic excitations can contribute substantially to the effective mass of the conduction electrons in rare-earth systems. These excitations are magnetic excitons in a paramagnetic system (e.g., praseodymium), and spin waves in magnetically ordered rare-earths. The mass enhancement appeared to be large, magnetic field dependent, and proportional to the static susceptibility of magnetic system. According to estimations of the electronic specific-heat coefficients, performed in Ref. [25], the corresponding effective masses increase in the series of heavy rare-earth metals. This trend correlates with the observed cyclotron masses in $\mathrm{ErGa}_{3}$ and $\mathrm{TmGa}_{3}$, but considerable work is needed to employ the theory developed in Ref. [25] for a quantitative description of cyclotron masses in magnetic $\mathrm{REGa}_{3}$ compounds. In this connection the results [26] of the dHvA effect studies at ambient pressure in isostructural $\mathrm{REIn}_{3}$ compounds, which possess similar FS's, should be taken into consideration as well.

\section{Summary}

As a whole, the calculated pressure derivatives of the dHvA frequencies in $\mathrm{ErGa}_{3}$ appeared to be in agreement with the experimental ones although the nature of some discrepancies is not clear. In particular, the difference between pressure derivatives of the spin-split FS cross-sectional areas is inconsistent with estimations based on the Stoner-Wohlfarth model, as well as with the results of $a b$ initio LSDA calculations. Also, a surprisingly large and intriguing pressure effect on cyclotron masses has been observed in $\mathrm{ErGa}_{3}$, which cannot be explained within the standard rare-earth model employed. It has to be emphasized that different interactions (exchange splitting, $\mathrm{CF}$ and magnetic-quadrupolar excitations, spin waves) have to be taken into account in a further theoretical analysis of the revealed pressure effects on the FS and cyclotron masses. In addition, more experimental data on the pressure dependence of the dHvA frequencies and cyclotron masses in $\mathrm{REGa}_{3}$ and $\mathrm{REIn}_{3}$ compounds are much needed to elucidate the nature of these effects

The authors dedicate this work to the 80th anniversary of B. I. Verkin, who was a pioneer of the dHvA effect studies under pressure [27].

We are grateful to J. Klamut, I. V. Svechkarev and B. Johansson for their kind support.

This work has been partly supported by the Royal Swedish Academy of Sciences.

1. V. B. Pluzhnikov, A. Czopnik, and I. V. Svechkarev, Physica 212, 375 (1995)

2. V. B. Pluzhnikov, A. Czopnik, G. E. Grechnev, N. V. Savchenko, and W. Suski, Phys. Rev. B59, 7893 (1999).

3. V. B. Pluzhnikov, A. Czopnik, and G. E. Grechnev, $J$. Phys.: Cond. Matter (1999), 11, 4507 (1999).

4. P. Morin, M. Giraud, P. L. Regnault, E. Roudaut, and A. Czopnik, J. Magn. Magn. Mater. 66, 345 (1987).

5. J. Pelleg, G. Kimmel, and D. Dayan, J. Less-Common Met. 81, 33 (1981).

6. T. F. Smith, C. W. Chu, and M. B. Maple, Cryogenics 9, 53 (1969).

7. K. R. Lea, M. J. M. Leask, and W. P. Wolf, J. Phys. Chem. Solids 23, 1381 (1962)

8. P. Morin, J. Rouchy, M. Giraud, and A. Czopnik, $J$ Magn. Magn. Mater. 67, 95 (1987).

9. A. Czopnik, A. S. Panfilov, and I. V. Svechkarev, Fiz. Nizk. Temp. 20, 48 (1994) [Low Temp. Phys. 20, 39 (1994)].

10. G. E. Grechnev, A. S. Panfilov, I. V. Svechkarev, K. H. J. Buschow, and A. Czopnik, J. Alloys Compd. 226, 107 (1995).

11. A. Murasik, A. Czopnik, L. Keller, and P. Fischer, J. Magn. Magn. Mater. (1999), in press.

12. M. S. S. Brooks and B. Johansson, in: Ferromagnetic Materials, vol. 7, K. H. J. Buschow (ed.), North-Holland, Amsterdam (1993) p. 139.

13. R. Ahuja, S. Auluck, B. Johansson, and M. S. S. Brooks, Phys. Rev. B50, 5147 (1994).

14. A. G. Petukhov, W. R. L. Lambrecht, and B. Segall, Phys. Rev. B53, 4324 (1996)

15. B. I. Min, H. J. F. Jansen, T. Oguchi, and A. J. Freeman, J. Magn. Magn. Mater. 61, 139 (1986).

16. J. F. Herbst and J. W. Wilkins, in: Handbook on the Physics and Chemistry of Rare Earths, vol. 10, K. A. Gschneidner, Jr, L. Eyring, and S. Hufner (eds.), NorthHolland, Amsterdam (1987), p. 321.

17. A. J. Freeman, B. I. Min, and M. R. Norman, in: Hand book on the Physics and Chemistry of Rare Earths, vol. 10, K. A. Gschneidner, Jr, L. Eyring, and S. Hufner (eds.), North-Holland, Amsterdam (1987), p. 165.

18. M. S. S. Brooks, L. Nordstrom, and B. Johansson, Physica B172, 95 (1991).

19. U. von Barth and L. Hedin, J. Phys. C5, 1629 (1972).

20. O. K. Andersen, Phys. Rev. B12, 3060 (1975).

21. H. L. Skriver, The LMTO Method, Springer, Berlin (1984). 
22. O. K. Andersen, O. Jepsen, and M. Sob, in: Electronic Band Structure and its Applications, M.Yussouff (ed.), Springer, Berlin (1987) p. 1.

23. G. E. Grechnev, A. S. Panfilov, I. V. Svechkarev, A. Delin, B. Johansson, J. M. Wills, and O. Eriksson, J. Magn. Magn. Mater. 192, 137 (1999).

24. G. Lonzarich and A. V. Gold, Can. J. Phys. 52, 694 (1974).

25. P. Fulde and J. Jensen, Phys. Rev. B27, 4085 (1983).
26. N. Nagai, I. Umehara, T. Ebihara, A. K. Albessard, H. Sugawara, T. Yamazaki, K. Satoh, and Y. Onuki, Physica B186-188, 139 (1993).

27. B. I. Verkin, I. M. Dmitrenko, and B. G. Lazarev, $Z h$. Eksp. Teor. Fiz. 31, 538 (1956) [Sov. Phys. JETP 4, 432 (1957)]; I. M. Dmitrenko, B. I. Verkin, and B. G. Lazarev, Zh. Eksp. Teor. Fiz. 33, 287 (1957) [Sov. Phys. JETP 6, 223 (1958)]; I. M. Dmitrenko, B. I. Verkin, and B. G. Lazarev, Zh. Eksp. Teor. Fiz. 35, 328 (1958) [Sov. Phys. JETP 8, 229 (1959)]. 